hep-th/0106237

\title{
Brane World in a Topological Black Hole Bulk
}

\author{
Danny Birmingham and Massimiliano Rinaldif \\ Department of Mathematical Physics \\ University College Dublin \\ Belfield, Dublin 4, Ireland
}

\begin{abstract}
We consider a static brane in the background of a topological black hole, in arbitrary dimensions. For hyperbolic horizons, we find a solution only when the black hole mass assumes its minimum negative value. In this case, the tension of the brane vanishes, and the brane position coincides with the location of the horizon. For an elliptic horizon, we show that the massless mode of Randall-Sundrum is recovered in the limit of large black hole mass.
\end{abstract}

July 2001

\footnotetext{
${ }^{1}$ E-mail: dannyb@pop3.ucd.ie

${ }^{2}$ Email: massimiliano.rinaldi@ucd.ie
} 


\section{Introduction}

The possibility that our universe could be modelled as a brane residing in a higher-dimensional anti-de Sitter spacetime has received renewed impetus recently [1, 2]. In this Randall-Sundrum scenario, the tension of the brane is fixed in terms of the bulk cosmological constant, so that the effective four-dimensional cosmological constant is zero. A key point in the analysis is that although the extra dimension is infinite in extent, the graviton fluctuations include a normalizable zero mode which is localized near the brane. In this way, one can recover the usual gravitational physics of four dimensions within a higher-dimensional context.

After these initial investigations, this scenario was generalized in several ways. In particular, a static brane in the background of a five-dimensional Schwarzschild-AdS black hole was studied in 3 , 4 . In this case, the tension and position of the brane are tuned in terms of the black hole mass and cosmological constant. The dynamics of a brane universe in a black hole background has also been considered, see 5, 6, 7], for example. Furthermore, the dynamics has been studied within the context of the AdS/CFT correspondence. One finds an interesting relation between the temperature and entropy of the black hole and the Hubble parameter as the brane crosses the horizon [8]. This analysis has also been extended to the topological black hole case [9, 10].

In this paper, we extend the discussion of a static brane to the case of a topological black hole bulk, in arbitrary dimensions. It is well known that anti-de Sitter space affords the possibility of black hole solutions, in which the topology of the horizon is non-spherical [1]]-[15]. The matching conditions at the brane fix both the brane tension and the brane position in terms of the black hole mass and cosmological constant [3, 4, 7]. The key point for the case of hyperbolic black holes is the existence of a negative mass spectrum. We find a brane world solution only when the black hole mass takes its minimum (negative) value. In this case, the tension of the brane vanishes, and the brane position coincides with the horizon. Unfortunately, the extent of the extra dimension is then zero. We also analyze the case of an elliptic horizon. In particular, it is shown that the original Randall-Sundrum graviton fluctuation equations are recovered in the limit of large black hole mass.

\section{The Model}

We consider the following action [1, 2],

$$
S=\int d^{d-1} x d z \sqrt{-G}\left(2 \mu^{d-2} R-\Lambda\right)+\int d^{d-1} x \sqrt{-g}(L-V)
$$

where $\mu$ is the $d$-dimensional Planck mass, $L$ the Lagrangian of the matter living on the brane, $V$ is the brane tension, $\Lambda$ is the $d$-dimensional cosmological constant, and $G_{M N}$ is the $d$ dimensional metric with indices $(M, N=0,1, \ldots,(d-1))$. The induced metric on the brane world is denoted by $g_{\mu \nu}$, and is given by $g_{\mu \nu}=G_{\mu \nu}\left(z=z_{b}\right)$, where $z_{b}$ is the position of the brane. The coordinates of the bulk spacetime are denoted by $x^{M}=\left(t, x^{i}, z\right)$, while the subset $x^{\mu}=\left(t, x^{i}\right)$ are the coordinates of the brane world. We shall consider the case with no matter 
on the brane, i.e., $L=0$. The corresponding equations of motion are given by

$$
R_{M N}-\frac{1}{2} G_{M N} R=-\frac{1}{4 \mu^{d-2}}\left[\Lambda G_{M N}+V \frac{\sqrt{-g}}{\sqrt{-G}} g_{\mu \nu} \delta_{M}^{\mu} \delta_{N}^{\nu} \delta\left(z-z_{b}\right)\right] .
$$

To begin, we consider the following ansatz for the bulk spacetime:

$$
d s^{2}=-\left(k+\frac{r^{2}}{l^{2}}-\frac{2 M}{r^{d-3}}\right) d t^{2}+\left(k+\frac{r^{2}}{l^{2}}-\frac{2 M}{r^{d-3}}\right)^{-1} d r^{2}+r^{2} \sigma_{i j}(x) d x^{i} d x^{j} .
$$

This metric corresponds to a $d$-dimensional topological black hole 15. In particular, the parameter $k$ classifies the horizon topology, with $k=-1,0,+1$ corresponding to hyperbolic, flat, and elliptic horizons, respectively. The five-dimensional case for $k=1$ was considered in [3, 4]. The parameter $M$ is identified with the mass of the black hole, and $l$ is the radius of anti-de Sitter space. In our conventions, the cosmological constant is given by $\Lambda=-2(d-1)(d-2) \mu^{d-2} / l^{2}$. Finally, $\sigma_{i j}$ is the metric on the horizon of the black hole, which we assume to be an Einstein space with curvature $k$.

To proceed, it is convenient to change variables [3], and consider

$$
d z=\frac{1}{\sqrt{k+\frac{r^{2}}{l^{2}}-\frac{2 M}{r^{d-3}}}} d r=\frac{1}{A(r)} d r .
$$

The metric then reads:

$$
d s^{2}=-A(z)^{2} d t^{2}+B(z)^{2} \sigma_{i j}(x) d x^{i} d x^{j}+d z^{2},
$$

where $A(z)$ is defined by (田), and $B(z)=r(z)$. With this ansatz, the non-vanishing components of the Ricci tensor are given by

$$
\begin{aligned}
R_{z z} & =-\frac{A^{\prime \prime}}{A}-(d-2) \frac{B^{\prime \prime}}{B} \\
R_{t t} & =A A^{\prime \prime}+(d-2) \frac{A A^{\prime} B^{\prime}}{B} \\
R_{i j} & =R_{i j}(\sigma)-\sigma_{i j}\left(B B^{\prime \prime}+\frac{B B^{\prime} A^{\prime}}{A}+(d-3) B^{\prime 2}\right) .
\end{aligned}
$$

Here, the prime denotes differentiation with respect to $z$, and $x^{i}$ with $(i=1, \ldots,(d-2))$ denote the spatial coordinates of the horizon. Since the horizon is an Einstein space, we have [15]

$$
R_{i j}(\sigma)=(d-3) k \sigma_{i j}
$$

Hence, the Ricci scalar curvature is given by

$$
R=(d-2)\left[(d-3) \frac{k}{B^{2}}-2 \frac{A^{\prime} B^{\prime}}{A B}-2 \frac{B^{\prime \prime}}{B}-(d-3) \frac{B^{\prime 2}}{B^{2}}\right]-2 \frac{A^{\prime \prime}}{A} .
$$


The $z z, t t$, and $i j$ equations of motion are given respectively by

$$
\begin{aligned}
0 & =-k+\left(\frac{2}{d-3}\right) \frac{A^{\prime} B^{\prime} B}{A}+B^{\prime 2}+\frac{\Lambda}{2 \mu^{d-2}} \frac{B^{2}}{(d-2)(d-3)}, \\
0 & =k-\frac{2}{(d-3)} B B^{\prime \prime}-B^{\prime 2}-\frac{B^{2}}{2 \mu^{d-2}(d-2)(d-3)}\left(\Lambda+V \delta\left(z-z_{b}\right)\right), \\
0 & =\frac{(d-3)}{2}\left[-(d-4) k+(d-4) B^{\prime 2}+2 B B^{\prime \prime}+2 \frac{A^{\prime} B^{\prime} B}{A}\right]+\frac{B^{2} A^{\prime \prime}}{A} \\
& +\frac{B^{2}}{4 \mu^{d-2}}\left(\Lambda+V \delta\left(z-z_{b}\right)\right) .
\end{aligned}
$$

One sees immediately that the latter two equations depend on the brane tension $V$. It is also straightforward to check that these equations hold in the bulk [15], when $V=0$.

\section{Matching Conditions}

In the original Randall-Sundrum model [1], the extra dimension is chosen to be periodic with an orbifold structure, corresponding to a space $S^{1} / \mathbf{Z}_{\mathbf{2}}$. In our case, we follow this suggestion by cutting off the extra coordinate at the horizon of the black hole $\left(z_{H}\right)$ and at the brane position $\left(z_{b}\right)$, see [3, 7]. The spacetime in between will then be referred to as the bulk. Then, by a similar orbifold procedure, we replace the cut part by a symmetric copy of the bulk. This can be explicitly performed by the change of variables [3], $\bar{z}=z_{b}-z$, with $z_{H} \leq z \leq z_{b}$, i.e., we consider only the spacetime between the horizon and the brane. Next, we identify $\bar{z} \rightarrow-\bar{z}$. The line element then becomes

$$
d s^{2}=-A(\bar{z})^{2} d t^{2}+B(\bar{z})^{2} \sigma_{i j}(x) d x^{i} d x^{j}+d \bar{z}^{2} .
$$

Note that, in these new coordinates, $\bar{z}=0$ corresponds to the brane position, while $\bar{z}_{H} \equiv z_{b}-z_{H}$ is the position of the horizon. The metric components are continuous at $\bar{z}=0$, but their first derivatives are not. Therefore, any second derivative carries a singular behaviour at the brane position. Thus,

$$
A^{\prime \prime}(|\bar{z}|)=A^{\prime \prime}(\bar{z})+2 A^{\prime}(\bar{z}) \delta(\bar{z})
$$

There is no matching condition at the location of the horizon, as follows by definition from (4).

Equations (10) and (11) then yield two conditions on the brane tension and brane position. This leads to a specification of $V$ and $r(0)$ in terms of the the black hole mass $M$, the cosmological constant $l$, and the topological label $k$. We find that

$$
V=8(d-2) \mu^{d-2} \sqrt{\frac{k}{r(0)^{2}}+\frac{1}{l^{2}}-\frac{2 M}{r(0)^{d-1}}},
$$

with the position of the brane given by

$$
r(0)^{d-3}=\frac{(d-1) M}{k} .
$$


By combining these, the brane tension can be expressed in the form

$$
V=8(d-2) \mu^{d-2}\left[\frac{1}{l^{2}}+(d-3)\left(\frac{k}{d-1}\right)^{\frac{d-1}{d-3}} \frac{1}{M^{\frac{2}{d-3}}}\right]^{1 / 2} .
$$

According to the three different topologies, we have the following scenario.

- For $k=-1$, the mass spectrum includes black holes with negative mass, $M \geq M_{\text {crit }}$, where 15]

$$
M_{\text {crit }}=-\frac{l^{d-3}}{d-1}\left(\frac{d-3}{d-1}\right)^{(d-3) / 2} .
$$

The corresponding horizon location is denoted by $r_{c r i t}$, and given by

$$
r_{\text {crit }}=l\left(\frac{d-3}{d-1}\right)^{1 / 2}
$$

One can re-write (16) in the form

$$
V=8(d-2) \frac{\mu^{d-2}}{l}\left[1-\left(\frac{M_{c r i t}}{M}\right)^{\frac{2}{d-3}}\right]^{1 / 2}
$$

Clearly, a real-valued brane position is possible by choosing $M<0$ in (15). However, it then follows from (19) that the only solution with real-valued brane tension corresponds to $M=M_{\text {crit }}$. Moreover, the brane tension then vanishes, and the position of the brane coincides with the location of the horizon $r(0)=r_{c r i t}$. Unfortunately, the range of the extra coordinate is also zero, and consequently this case becomes degenerate.

- For $k=0$, the only solution is when $M=0$. In this case, we recover the original Randall-Sundrum model [2] for $d=5$.

- For $k=1$, we have a range of solutions depending on the mass of the black hole. The brane tension is the same as that found in the original Randall-Sundrum model [1, 2], plus a correction proportional to an inverse power of the mass. Therefore, in the zero mass limit the tension diverges, while in the large $M$ limit we recover the Randall-Sundrum brane tension. In this large $M$ limit, the brane runs off to infinity faster than the horizon. For example, in five dimensions [⿴囗⿰丿㇄口], one has $r(0) \propto M^{1 / 2}$ and $r_{H} \propto M^{1 / 4}$. Therefore, an observer living on the brane will find himself in the asymptotic region of the black hole.

\section{Perturbations}

In this section, we analyze the graviton propagation for the $k=1$ case [3]. In particular, we show that the massless mode of Randall-Sundrum is recovered in the large mass limit. We first 
note that eqn. (21) can written in the form

$$
R_{M N}=-\frac{(d-1)}{l^{2}} G_{M N}+T_{M N}
$$

where

$$
T_{M N}=\frac{V}{4 \mu^{d-2}} \frac{\sqrt{-g}}{\sqrt{-G}} \delta\left(z-z_{b}\right)\left[\frac{(d-1)}{(d-2)} G_{M N}-g_{\mu \nu} \delta_{M}^{\mu} \delta_{N}^{\nu}\right] .
$$

If we introduce a small perturbation $h_{M N}\left(z, x^{\mu}\right)$, with $h_{z \mu}=h_{z z}=0$, then eqn. (20) reduces to

$$
R_{M N}^{(1)}=-\frac{(d-1)}{l^{2}} h_{M N}+T_{M N}^{(1)},
$$

with

$$
R_{M N}^{(1)}=\frac{1}{2}\left(-\nabla_{M} \nabla_{N} h-\nabla^{2} h_{M N}+\nabla^{A} \nabla_{M} h_{N A}+\nabla^{A} \nabla_{N} h_{M A}\right),
$$

and

$$
T_{M N}^{(1)}=\frac{V}{4(d-2) \mu^{d-2}} \delta\left(z-z_{b}\right) h_{M N} .
$$

By commuting the covariant derivatives in (23), and imposing the Lorentz gauge $\nabla^{M} h_{M N}=0$, we find that the perturbation equation (22) becomes

$$
\nabla_{M} \nabla_{N} h+\nabla^{2} h_{M N}-R_{A M N B} h^{A B}-R_{A N M B} h^{A B}=0 .
$$

In our case, the spacetime is warped asymmetric, with $A(z)$ different from $B(z)$. If we assume that the perturbation is traceless, $h^{M}{ }_{M}=0$, then the $z z$, $z t$, and $z i$ equations are given respectively by

$$
\begin{aligned}
\left(\frac{B^{\prime 2}}{B^{2}}-\frac{B^{\prime \prime}}{B}\right) h_{l}^{l}+\left(\frac{A^{\prime 2}}{A^{2}}-\frac{A^{\prime \prime}}{A}\right) h_{t}^{t}=0 \\
\left(\frac{A^{\prime}}{A}-\frac{B^{\prime}}{B}\right) \partial_{t} h_{t t}=0, \quad\left(\frac{A^{\prime}}{A}-\frac{B^{\prime}}{B}\right) \partial_{t} h_{t i}=0 .
\end{aligned}
$$

Furthermore, the remaining $t t, t i$, and $i j$ equations are of the form

$$
\begin{aligned}
0 & =\left[\partial_{z}^{2}+G^{t t} \partial_{t}^{2}+G^{i j} \nabla_{i} \nabla_{j}+\left((d-2) \frac{B^{\prime}}{B}-3 \frac{A^{\prime}}{A}\right) \partial_{z}-2 \frac{A^{\prime \prime}}{A}+2 \frac{A^{\prime 2}}{A^{2}}-2(d-3) \frac{A^{\prime} B^{\prime}}{A B}\right] h_{t t}, \\
0 & =\left[\partial_{z}^{2}+G^{t t} \partial_{t}^{2}+G^{j k} \nabla_{j} \nabla_{k}+\left((d-4) \frac{B^{\prime}}{B}-\frac{A^{\prime}}{A}\right) \partial_{z}-\frac{A^{\prime \prime}}{A}-\frac{B^{\prime \prime}}{B}-(d-5) \frac{A^{\prime} B^{\prime}}{A B}\right. \\
& \left.-(d-3) \frac{B^{\prime 2}}{B^{2}}\right] h_{t i}, \\
0 & =\left[\partial_{z}^{2}+G^{t t} \partial_{t}^{2}+G^{k l} \nabla_{k} \nabla_{l}+\left(\frac{A^{\prime}}{A}+(d-6) \frac{B^{\prime}}{B}\right) \partial_{z}-2 \frac{B^{\prime \prime}}{B}-2 \frac{A^{\prime} B^{\prime}}{A B}-2(d-5) \frac{B^{\prime 2}}{B^{2}}\right. \\
& \left.-\frac{2}{B^{2}}\right] h_{i j}-2\left(\frac{B^{\prime 2}}{B^{2}}-\frac{A^{\prime} B^{\prime}}{A B}-\frac{1}{B^{2}}\right) h_{l}^{l} G_{i j} .
\end{aligned}
$$


For the symmetric case, $A(z)=B(z)$, these equations agree with [16].

One can now examine these equations in the near-brane region, by expanding the coefficients around the brane position. In the limit of large black hole mass, the fluctuation equations reduce to the single equation

$$
\left[\partial_{z}^{2}+G^{t t} \partial_{t}^{2}+G^{i j} \nabla_{i} \nabla_{j}-(d-5) \frac{1}{l} \partial_{z}-2(d-3) \frac{1}{l^{2}}+\frac{4}{l} \delta\left(z-z_{b}\right)\right] h_{\mu \nu}=0 .
$$

It is instructive to analyze the five-dimensional case in more detail. The gravitational equations on the 3-brane have been studied in [17], see also [18. In particular, the fourdimensional effective vacuum energy (in 4-dimensional Planck units), and the effective Planck mass are given by [7, 17]

$$
\Lambda_{4}=\frac{3 k^{2}}{8 M}, \quad M_{P L}^{2}=\mu^{3} l\left(1+\frac{l^{2}}{8 M}\right)^{-\frac{1}{2}} .
$$

Thus, we see that $\Lambda_{4}$ tends to zero for large mass, while $M_{P L}^{2}$ tends to $\mu^{3} l$. The latter corresponds to the Randall-Sundrum value [2] of the Planck mass, when the compactification radius is infinite. In this sense, one can regard the black hole mass parameter as being analogous to the compactification radius of the Randall-Sundrum model. Since $\Lambda_{4}$ vanishes in the large mass limit, we see that eqn. (28) does indeed coincide with the Randall-Sundrum equation for graviton fluctuations.

In conclusion, we have considered a static brane in the background of a $d$-dimensional topological black hole. One finds that both the tension and position of the brane are fixed by the matching conditions in Einstein's equations. For the case of $k=-1$, there is a solution only when the black hole mass takes its lowest (negative) value. However, since the brane is located at the horizon, the extra dimension does not have extent. For the $k=1$ case, it is in the limit of large black hole mass that one recovers the original Randall-Sundrum model, with a massless localized graviton. Aspects of brane worlds in black hole backgrounds of higher derivative gravity have been studied in [19, 20]. Recently, various brane solutions in charged dilatonic backgrounds have been discussed, and the $k=0$ and $k=-1$ solutions have particularly interesting properties [21].

\section{Acknowledgements}

The research of M.R. is supported by Enterprise Ireland grant BR/1999/031, and P.E. 2000/2001 from Bologna University. M.R. is grateful to P.J. Silva for valuable correspondence, and to R. Balbinot for discussions.

\section{References}

[1] L. Randall and R. Sundrum, Phys. Rev. Lett. 83 (1999) 3370; hep-ph/9905221.

[2] L. Randall and R. Sundrum, Phys. Rev. Lett. 83 (1999) 4690; hep-th/9906064. 
[3] C. Gomez, B. Janssen and P.J. Silva, JHEP 0004 (2000) 027; hep-th/0003002.

[4] A. Kamenshchik, U. Moschella, and V. Pasquier, Phys. Lett. B487 (2000) 7; gr-qc/0005011.

[5] P. Kraus, JHEP 9912 (1999) 011; hep-th/9910149.

[6] H. Stoica, S.-H. Henry Tye, and I. Wasserman, Phys. Lett. B482 (2000) 205; hepth/0004126.

[7] C. Csaki, J. Erlich and C. Grojean, Nucl. Phys. B604 (2001) 312; hep-th/0012143.

[8] I. Savonije and E. Verlinde, Phys. Lett. B507 (2001) 305; hep-th/0102042.

[9] R.-G. Cai, Phys. Rev. D63 (2001) 124018; hep-th/0102113.

[10] D. Youm, Phys. Lett. B515 (2001) 170; hep-th/0105093.

[11] S. Åminneborg, I. Bengtsson, S. Holst, and P. Peldán, Class. Quantum Grav. 13 (1999) 2707 ; gr-qc/9604005.

[12] R.B. Mann, Class. Quantum Grav. 14 (1997) L109; gr-qc/9607071.

[13] L. Vanzo, Phys. Rev. D56 (1997) 6475; gr-qc/9705004.

[14] D.R. Brill, J. Louko, and P. Peldán, Phys. Rev. D56 (1997) 3600; gr-qc/9705012.

[15] D. Birmingham, Class. Quantum Grav. 16 (1999) 1197; hep-th/9808032.

[16] N. Alonso-Alberca, P. Meessen and T. Ortin, Phys. Lett. B482 (2000) 400; hep-th/0003248.

[17] T. Shiromizu, K. Maeda and M. Sasaki, Phys. Rev. D62 (2000) 024012; gr-qc/9910076.

[18] N. Deruelle and J. Katz, Gravity on Branes, gr-qc/0104007.

[19] S. Nojiri and S.D. Odintsov, Phys. Lett. B493 (2000) 153; hep-th/0007205.

[20] S. Nojiri, S.D. Odintsov, and S. Ogushi, Holographic Entropy and Brane FRW-dynamics from AdS Black Hole in 45 Higher Derivative Gravity, hep-th/0105117.

[21] C. Grojean, F. Quevedo, G. Tasinato, and I. Zavala, JHEP 0108 (2001) 005; hepth/0106120. 